\title{
Line of sight depth of the Large and Small Magellanic Clouds
}

\author{
Annapurni Subramaniam and Smitha Subramaniam \\ Indian Institute of Astrophysics, Sarjapur Road, Koramangala II Block, \\ Bangalore -560034, India \\ email: purni@iiap.res.in, smitha@iiap.res.in
}

\begin{abstract}
We used the red clump stars from the Optical Gravitational Lensing Experiment (OGLE II) survey and the Magellanic Cloud Photometric Survey (MCPS), to estimate the line-of-sight depth. The observed dispersion in the magnitude and colour distribution of red clump stars is used to estimate the line-of-sight depth, after correcting for the contribution due to other effects. This dispersion due to depth, has a range from minimum dispersion that can be estimated, to $0.46 \mathrm{mag}$ (a depth of $500 \mathrm{pc}$ to $10.44 \mathrm{kpc}$ ), in the LMC. In the case of the $\mathrm{SMC}$, the dispersion ranges from minimum dispersion to 0.35 magnitude (a depth of $665 \mathrm{pc}$ to $9.53 \mathrm{kpc}$ ). The thickness profile of the LMC bar indicates that it is flared. The average depth in the bar region is $4.0 \pm 1.4 \mathrm{kpc}$. The halo of the LMC (using RR Lyrae stars) is found to have larger depth compared to the disk/bar, which supports the presence of an inner halo for the LMC. The large depth estimated for the LMC bar and the disk suggests that the LMC might have had minor mergers. In the case of the SMC, the bar depth $(4.90 \pm 1.23 \mathrm{kpc})$ and the disk depth $(4.23 \pm 1.48 \mathrm{kpc})$ are found to be within the standard deviations. We find evidence for an increase in depth near the optical center (up to $9 \mathrm{kpc}$ ). On the other hand, the estimated depth for the halo (RR Lyrae stars) and disk (RC stars) for the bar region of the SMC is found to be similar. Thus, increased depth and enhanced stellar as well as H I density near the optical center suggests that the SMC may have a bulge.
\end{abstract}

Keywords. stars: horizontal-branch, galaxies: bulges, galaxies: halos, Magellanic Clouds, galaxies: stellar content, galaxies: structure

\section{Introduction}

The Magellanic Clouds were believed to have interactions with our Galaxy as well as between each other (Westerlund 1997). The N-body simulations by Weinberg (2000) predicted that the LMC's evolution is significantly affected by its interactions with the Milky Way and the tidal forces will thicken and warp the LMC disk. Alves \& Nelson (2000) studied the carbon star kinematics and found that the scale height, h, increases from 0.3 to $1.6 \mathrm{kpc}$ over the range of radial distance, $\mathrm{R}$, from 0.5 to $5.6 \mathrm{kpc}$ and hence concluded that the LMC disk is flared. Using an expanded sample of carbon stars van der Marel et al. (2002) also found that the thickness of LMC disk increases with the radius. There has not been any direct estimate of the thickness or the line-of-sight depth of the bar and disk of the L\&SMC so far.

Mathewson, Ford \& Visvanathan (1986) found that SMC Cepheids extend from 43 to $75 \mathrm{kpc}$ with most Cepheids found in the neighbourhood of $59 \mathrm{kpc}$. Later, the line-of-sight depth of the SMC was estimated (Welch et al. 1987) by investigating the line-of-sight distribution and period-luminosity relation of Cepheids and found the line-of-sight depth of the SMC to be around $3.3 \mathrm{kpc}$. Hatzidimitriou \& Hawkins (1989), estimated the lineof-sight depth in the outer regions of the SMC to be around $10-20 \mathrm{kpc}$. Measurement 
of the thickness in the central regions of the Magellanic Clouds, especially the LMC, is of strong interest to understand the contribution of LMC's self lensing to the observed microlensing events from this Galaxy.

Red Clump (RC) stars are core helium burning stars which are metal rich and slightly more massive counter parts of horizontal branch stars. In this paper, we use the dispersions in the colour and magnitude distribution of RC stars for the depth estimation. The dispersion in colour is due to a combination of observational error, internal reddening (reddening within the disk of the LMC/SMC) and population effects. The dispersion in magnitude is due to internal disk extinction, depth of the distribution, population effects and photometric errors associated with the observations. By deconvolving other effects from the dispersion of magnitude, we can estimate the depth of the disk. The advantage of choosing RC stars as proxy is that there are large numbers of these stars available to determine the dispersions in their distributions with good statistics.

\section{Data}

Data for the LMC are taken from the OGLE II catalogue (Udalski et al. 2000). The average photometric error of red clump stars in I and V bands are around 0.05 magnitude. Photometric data with an error less than $0.15 \mathrm{mag}$ are considered for the analysis. The 26 strips of LMC are divided into 1664 regions. (V-I) vs. I CMDs are plotted for each region and red clump stars $(\geqslant 1000$ stars) were identified. For all the regions, red clump stars are well within a box in the CMD with boundaries $0.65-1.35$ mag in $(\mathrm{V}-\mathrm{I})$ colour and 17.5-19.5 mag in I magnitude. The OGLE data suffer from incompleteness due to crowding effects and it is corrected using the data given in Udalski et al. (2000).

The data for the SMC are taken from two surveys (OGLE II: Udalski et al. 1998, and MCPS: Zaritsky et al. 2002). The OGLE and MCPS photometric data with an error less than $0.15 \mathrm{mag}$ are considered for the analysis. The observed regions of the SMC OGLE and MCPS data are divided into 176 and 876 sub regions respectively. In the MCPS data the regions away from the bar are less dense compared to the bar region and out of 876 regions only 755 regions which have a reasonable number of stars $(\sim 1000$ stars $)$ are considered for the analysis. For all the regions, red clump stars are identified in the same region in the CMD with boundaries 0.65-1.35 mag in (V-I) colour and 17.5-19.5 mag in I magnitude. OGLE data are corrected for incompleteness.

\section{Analysis}

A spread in magnitude and colour of red clump stars is observed in the CMDs of both the LMC and SMC. The number distribution profile against colour and magnitude roughly resembles a Gaussian. The width of the Gaussian in the distribution of colour and magnitude is obtained using a non-linear least-squares method to fit the profile.

The RC stars in the disk of the LMC/SMC constitute a heterogeneous population spanning a range in mass, age and metallicity. The density of stars in various locations will also vary with star formation rate as a function of time. Girardi \& Salaris (2001) simulated the RC stars in the LMC using the star formation rate results from Holtzman et al. (1999) and the age-metallicity relation from Pagel \& Tautvaisiene (1998). They also simulated the RC stars in the SMC using the star formation results and age-metallicity relation from Pagel \& Tautvaisiene (1998). The intrinsic dispersions obtained from the above model are used in our estimation to account for the population effects. The estimated intrinsic dispersions in magnitude and colour distributions according to the theoretical 
model for the LMC are 0.1 and 0.025 mag respectively. In the case of the SMC, the values are 0.076 and $0.03 \mathrm{mag}$ respectively.

The average photometric errors of I and V band magnitudes are calculated for each region and the errors in I and (V-I) colour are estimated. These are subtracted from the observed width of the magnitude and colour distribution respectively. After correcting for population effects and the observational error in colour, the remaining spread in colour distribution is taken as due to the internal reddening, $\mathrm{E}(\mathrm{V}-\mathrm{I})$. This is converted into extinction in I band using the relation $A(I)=0.934 E(V-I)$, where $\mathrm{E}(\mathrm{V}-\mathrm{I})$ is the internal reddening value estimated for each location. The above relation is derived from the relations $E(V-I)=1.6 E(B-V)$ and $A(I)=0.482 A(V)$ (Rieke \& Lebofsky 1985).

The following relations are used for estimating the resultant dispersion due to depth:

$$
\begin{gathered}
\sigma_{\text {mag }}^{2}=\sigma_{\text {depth }}^{2}+\sigma_{\text {internal extinction }}^{2}+\sigma_{\text {intrinsic }}^{2}+\sigma_{\text {error }}^{2}, \\
\sigma_{\text {col }}^{2}=\sigma_{\text {internal reddening }}^{2}+\sigma_{\text {intrinsic }}^{2}+\sigma_{\text {error }}^{2} .
\end{gathered}
$$

\section{Results: LMC}

A two dimensional plot of the depth for the 1528 regions in the LMC is shown in figure 1. The optical center of the LMC is taken to be RA $=5^{\mathrm{h}} 19^{\mathrm{m}} 38^{\mathrm{s}}$, Dec $=-69^{\circ} 27^{\prime} 5^{\prime \prime} .2$ (J2000, de Vaucouleurs \& Freeman 1973). The plot shows a range of dispersion values from 0.033 to $0.46 \mathrm{mag}$ (a depth of $700 \mathrm{pc}$ to $10.5 \mathrm{kpc}$; average: $3.95 \pm 1.42 \mathrm{kpc}$ ) for the LMC central bar region. For the N-W disk region, the dispersion estimated ranges from minimal dispersion that can be estimated (limited by errors), to $0.33 \mathrm{mag}$ (a depth of $500 \mathrm{pc}$ to $7.7 \mathrm{kpc}$; average: $3.56 \pm 1.04 \mathrm{kpc}$ ). Regions in the bar between RA $80-84$ degrees show a reduced depth $(0.5-4 \mathrm{kpc}$, as indicated by yellow and black points). The regions to the east and west of the above region are found to have a larger depth $(2.0-$ $8.0 \mathrm{kpc}$, black, red and green points). Thus, the depth of the bar at its ends is larger than that near its center. The N-W region has a depth similar to the central region of the bar. In general, a thicker and heated up bar could be considered as a signature of minor mergers. Thus, the LMC is likely to have experienced minor mergers in its history.

Subramaniam (2006) studied the distribution of RR Lyrae in the bar region of the LMC. She found that the RR Lyrae stars in the bar region have a disk like distribution, but halo like location. The RR Lyrae stars are in the same evolutionary state as the RC stars, except that the RR Lyrae stars belong to an older and metal poor population and hence a proxy for the halo as it belongs to the Population II stars. We used the total depth estimated for RR Lyrae stars and compared it with the RC depth which can be considered as proxies for halo and disk respectively. It was seen that the depth estimated from RR Lyrae stars ranged between $4.0-8.0 \mathrm{kpc}$, suggesting that the RR Lyrae stars span a larger depth than the RC stars. Thus, at least in the central region of the LMC, the halo, as delineated by the RR Lyrae stars has a much larger depth than the disk, as delineated by the RC stars. This supports the idea of an inner halo for the LMC.

\section{Results: SMC}

Colour-coded two-dimensional plots of thickness for the two data sets are shown in figure 2 (OGLE data in the lower panel and MCPS data in the upper panel). The optical center of the SMC is taken to be RA $=00^{\mathrm{h}} 52^{\mathrm{m}} 12.5^{\mathrm{s}}$, Dec $=-72^{\circ} 49^{\prime} 43^{\prime \prime}$ (J2000, de Vaucouleurs \& Freeman 1973). The prominent feature in both plots is the presence of 


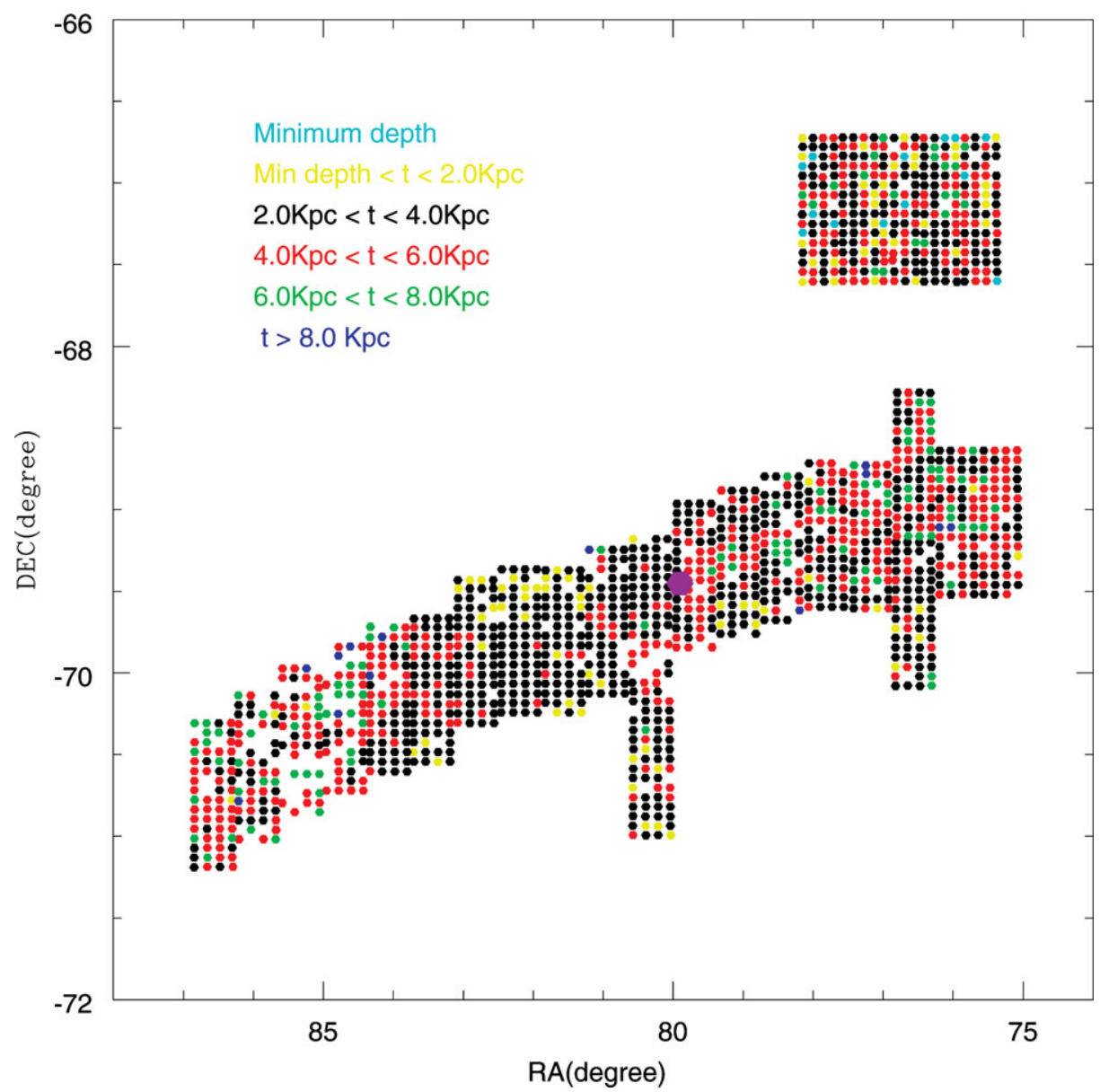

Figure 1. The line-of-sight depth in the bar region of the LMC. The colour codes used to denote the depth are explained. The optical center is shown as a purple dot.

blue and green points indicating increased depth, for regions located near the SMC optical center $(\sim 9 \mathrm{kpc})$. The net dispersions range from 0.10 to $0.35 \mathrm{mag}$ (corresponding to a depth of $2.8 \mathrm{kpc}$ to $9.6 \mathrm{kpc}$ ) in the OGLE data set and from minimum dispersion to $0.34 \mathrm{mag}$ (corresponding to a depth of $665 \mathrm{pc}$ to $9.47 \mathrm{kpc}$ ) in the MCPS data set. The average value of the SMC thickness estimated using the OGLE data set in the central bar region is $4.9 \pm 1.2 \mathrm{kpc}$ and the average thickness estimated using the MCPS data set, which covers a larger area than the OGLE data, is $4.42 \pm 1.46 \mathrm{kpc}$. The average depth obtained for the bar region alone is $4.97 \pm 1.28 \mathrm{kpc}$, which is very similar to the value obtained from the OGLE data. The depth estimated for the disk alone is $4.23 \pm 1.47 \mathrm{kpc}$. Thus the disk and the bar of the SMC do not show any significant difference in the depth. Evidence of increased depth in the outer regions of the disk is also indicated, this might suggest a large depth for the outer SMC. The enhanced central depth is shown in the averaged width over a limited RA and Dec in figure 3, using the MCPS data. The increased depth near the center is clearly indicated. Thus, the depth near the center is about $9.6 \mathrm{kpc}$, which is twice the average depth of the bar region $(4.9 \mathrm{kpc})$. The depth profile, especially the one along RA, is very similar to the luminosity profile of bulges and hence suggests the presence of a bulge in the SMC. 

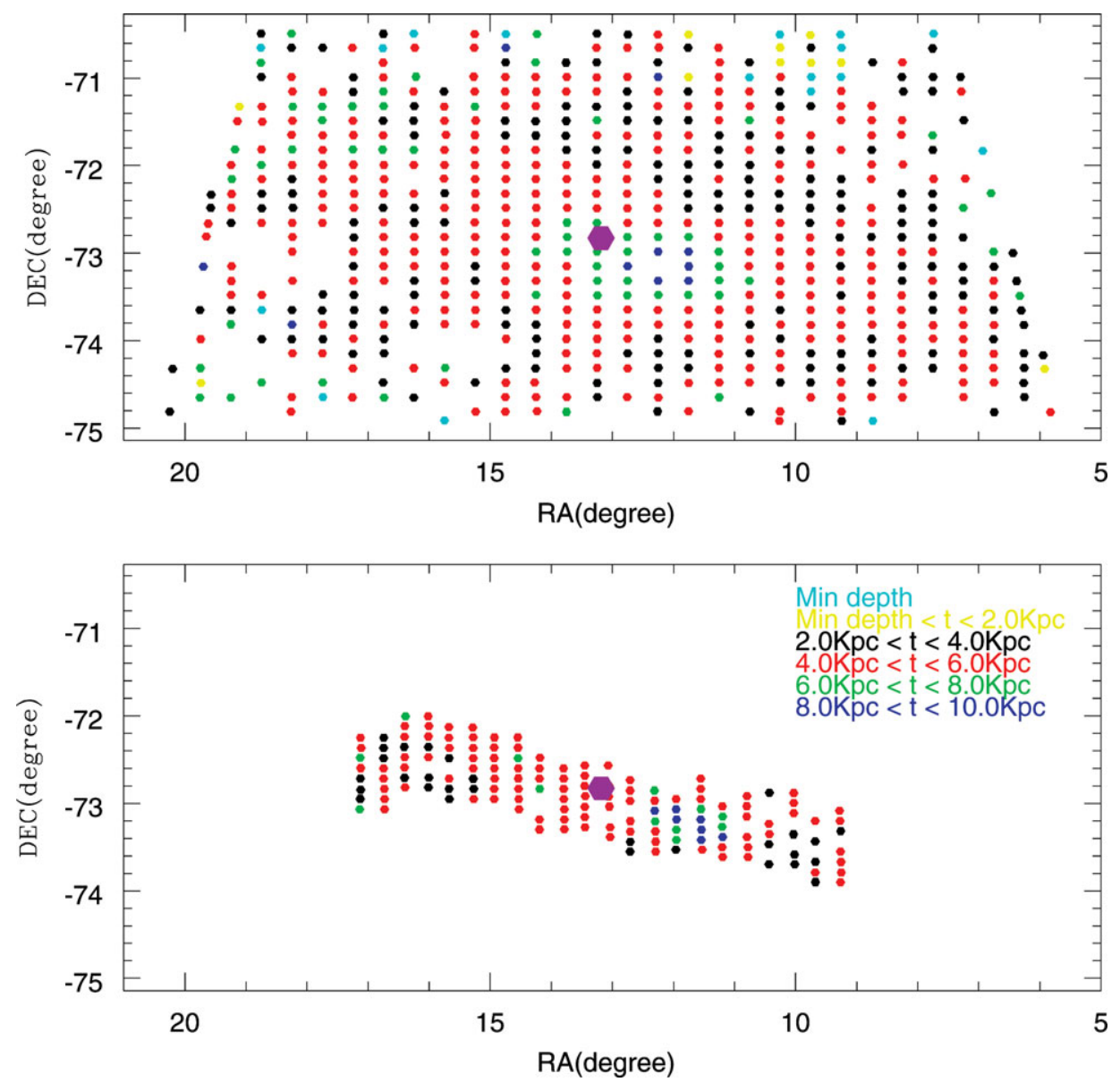

Figure 2. The line-of-sight depth in the bar and disk region of the SMC. The upper panel shows the depth derived from the MCPS data and the lower panel shows that derived from the OGLE data. The optical center is shown as a purple dot.

A comparison can be made between the halo and disk/bar of the SMC. The RR Lyrae stars from the OGLE II data were analysed similar to the procedure adopted by Subramaniam (2006) and the dispersion due to depth alone was estimated. In contrast to what is seen in the case of the LMC, both populations show a very similar dispersion in the SMC. The comparison not only suggests that the RR Lyrae stars and the RC stars occupy similar depth, but also indicates that they show a similar depth profile across the bar. The increased depth near the optical center is also closely matched. This suggests that the RR Lyrae stars and RC stars are born in the same location and occupy similar volume in the galaxy. This co-existence and the similar depth of RR Lyrae stars and the RC stars in the central region of the SMC can be easily explained, if it is the bulge. We also find that the old and young stellar density as well as the H I density show peaks near the region with bulge like depth. This supports the idea that the central region of the SMC could be its bulge. The elongation and the rather non-spherical appearance of the bulge could be due to tidal effects or minor mergers (Bekki \& Chiba 2008). 

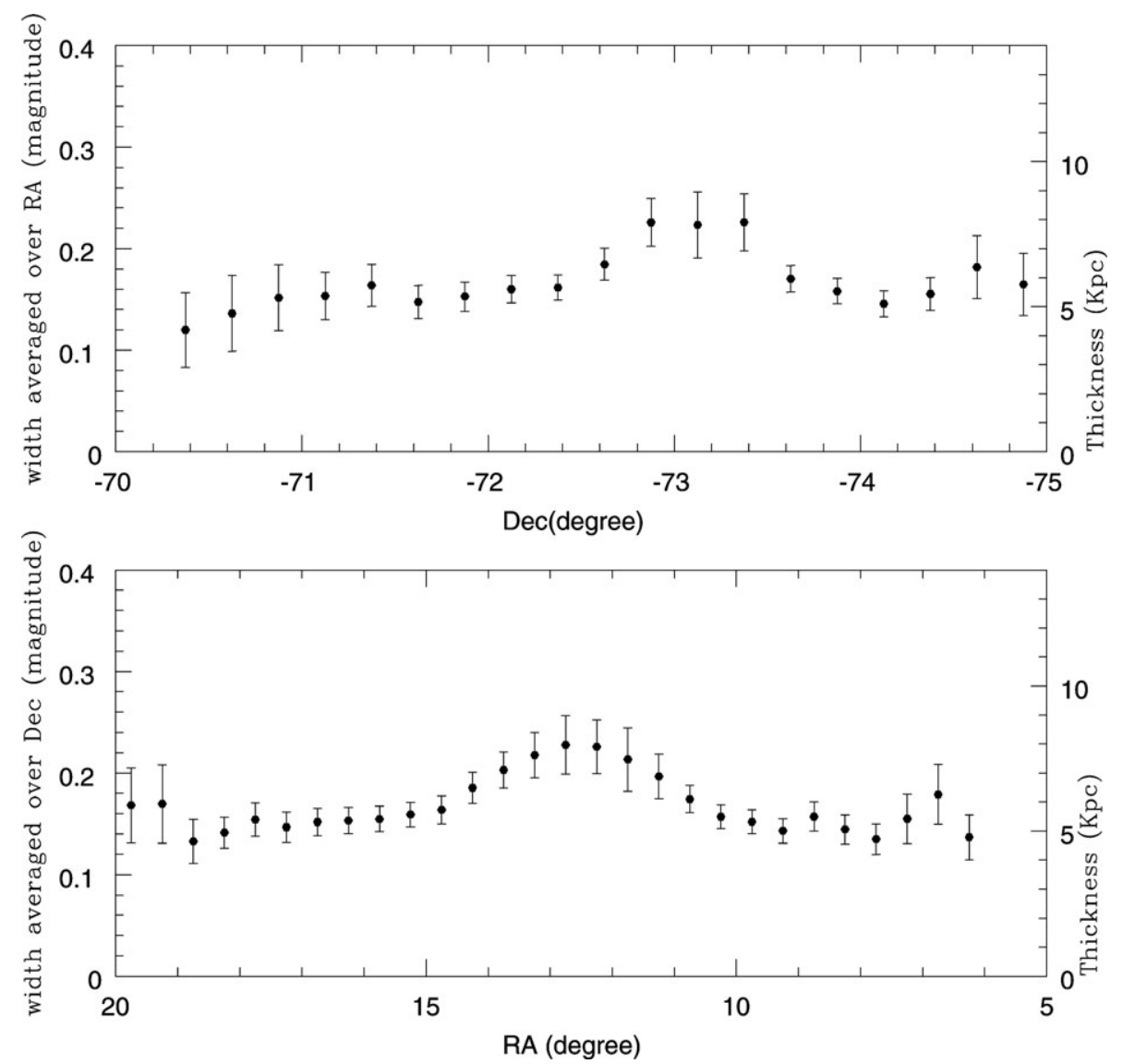

Figure 3. The line-of-sight depth averaged over a narrow range of RA and Dec near the center using the MCPS data. The errors correspond to the standard deviation over the area averaged.

\section{References}

Alves, D. R. \& Nelson, C. A. 2000, ApJ, 542, 789

Bekki, K. \& Chiba, M. 2008, ApJ, 679, L89

de Vaucouleurs, G. \& Freeman, K. C. 1973, Vistas Astron., 14, 163

Girardi, L. \& Salaris, M. 2001, MNRAS, 323, 109

Hatzidimitriou, D. \& Hawkins, M. R. S. 1989, MNRAS, 241, 667

Holtzman, J. A., Gallagher, J. S., III, Cole, A. A., et al. 1999, AJ, 118, 2262

Mathewson, D. S., Ford, V. L., \& Visvanathan, N. 1986, ApJ, 301, 664

Pagel, B. E. J. \& Tautvaisiene, G. 1998, MNRAS, 299, 535

Rieke, G. H. \& Lebofsky, M. J. 1985, ApJ, 288, 618

Subramaniam, A. 2006, A\&\&A, 449, 101

Udalski, A., Szymański, M., Kubiak, M., et al. 1998, AcA, 48,147 (SMC OGLE II data) Udalski, A., Szymański, M., Kubiak, M., et al. 2000, AcA, 50,307 (LMC OGLE II data) van der Marel, R. P., Alves, D. R., Hardy, E., \& Suntzeff, N. B. 2002, AJ, 124, 2639 Weinberg, M. D. 2000, ApJ, 532, 922

Welch, D. L., McLaren, R. A., Madore, B. F., \& McAlarey, C. W. 1987, ApJ, 321, 162

Westurlund, B. E. 1997, The Magellanic Clouds (Cambridge: CUP)

Zaritsky, D., Harris, J., Thompson, I. B., Grebel, E. K., \& Massey, P. 2002, AJ, 123, 855 